International Journal of Biomedicine | June 2021 - Volume 11, Issue Suppl_1: Abstracts from the Third Russian International Conference "Cryo-electron microscopy 2021: achievements and prospects"

ORAL ABSTRACT PRESENTATIONS

SESSION TITLE: STRUCTURE OF MEMBRANE PROTEINS

DOI: 10.21103/IJBM.11.Suppl_1.OR2

\title{
Abstract OR-2: The Formation of Dps-DNA Complexes under Different Conditions According to Cryo-EM and SAXS
}

$\underline{\text { Roman Kamyshinsky }}^{1,2}$, Yury Chesnokov ${ }^{1,2}$, Liubov Dadinova ${ }^{2}$, Andrey Mozhaev $^{2,3}$, Alexander Vasiliev ${ }^{1,2}$, Eleonora Shtykova ${ }^{2}$

\author{
${ }^{1}$ National Research Center "Kurchatov Institute," Moscow, Russia \\ ${ }^{2}$ Shubnikov Institute of Crystallography of FSRC "Crystallography and \\ Photonics" RAS, Moscow, Russia \\ ${ }^{3}$ Shemyakin-Ovchinnikov Institute of bioorganic chemistry of RAS, \\ Moscow, Russia
}

Background: The effect of Dps-DNA co-crystals formation, which occurs in stressed Escherichia coli cells exposed to extreme conditions, is well described in the literature. However, the exact mechanisms of co-crystals formation are yet to be postulated remaining largely unknown. Here we summarize the results obtained by our group over the last few years using cryo-Electron Microscopy (cryo-EM) and Small Angle X-ray Scattering (SAXS).

Methods: Samples for cryo-EM were plunge frozen in liquid ethane with Vitrobot Mark IV and studied with Titan Krios (ThermoFisher Scientific, US) cryo-EM, equipped with Falcon 2 direct electron detector, Image corrector (CEOS, Germany), and Volta phase plate.

Single Particle Analysis (SPA) and cryo-Electron Tomography (cryo-ET) studies were conducted with $300 \mathrm{kV}$ accelerating voltage in low dose mode using EPU and Tomography software (ThermoFisher Scientific, US). Cryo-EM data processing was conducted using Warp, CryoSPARC, IMOD, EMAN, and Relion software packages. SAXS measurements were performed at the EMBL on the P12 BioSAXS beam line at the PETRAIII storage ring (DESY, Hamburg).

Results: In this work, Dps-DNA complex formation is thoroughly studied using complementary cryo-EM (including SPA, cryo-ET, and subtomogram averaging) and SAXS methods. The formation of individual complexes of Dps with small linear DNA fragments and the Dps-Dps interaction was visualized 
using cryo-EM. It was found that Dps-DNA complex remains stable under various conditions and while the addition of different ions leads to the disruption of co-crystals, the process is completely or partially reversible.

Conclusion: Recent studies conducted by our group showed that Dps-DNA cocrystals adopt triclinic or cubic crystal lattice (FEBS Lett., 2019; Biomolecules, 2020). Here we present the results on the studies of Dps interaction with small linear DNA fragments, demonstrate the effects of $\mathrm{MgCl}_{2}, \mathrm{FeSO}_{4}$, and EDTA on the Dps-DNA complex and individual Dps protein structure, discuss the influence of the temperature and time on the co-crystals.

\section{Key Words: Dps-DNA • co-crystals $\bullet$ cryo-EM $\bullet$ SAXS}

This work was supported by the Russian Science Foundation (project № 1874-10071)

*Corresponding author: Roman Kamyshinsky. E-mail: kamyshinsky.roman@gmail.com

International Journal of Biomedicine. 2021;11 Suppl 1: S7.

doi: 10.21103/IJBM.11.Suppl_1.0R2

(C)2021 International Medical Research and Development Corporation 